\title{
La economía política de las transferencias fiscales a los gobiernos regionales del Perú
}

\author{
Leonardo E. Letelier S. y Gonzalo Neyra A.
}

En este trabajo se explora el modelo peruano de asignación de recursos ordinarios otorgados a los gobiernos regionales como transferencias fiscales discrecionales del gobierno nacional. Se estimó un modelo empírico basado en un panel de datos anuales correspondientes al período 2004-2010. Si bien las transferencias nacionales están considerablemente sesgadas hacia las regiones que prestaron menor apoyo al gobierno nacional en las elecciones, los datos presentados sugieren que este efecto es más significativo en el comienzo del mandato gubernamental. Sin embargo, a largo plazo, las regiones opositoras parecen concentrar a electores más volátiles, lo que es compatible con la hipótesis del "votante bisagra". Resulta interesante que las regiones que apoyaron fervientemente al presidente reciban muy pocos beneficios. Por último, los conflictos regionales, el efecto de los cabildeos de grupos civiles organizados a nivel regional y el tamaño del distrito electoral, entre otras variables, son factores estadísticamente significativos en las estimaciones. 


\section{I}

\section{Introducción}

En el presente artículo se explora la dinámica de las transferencias fiscales a los gobiernos regionales en el Perú, y se proporcionan pruebas empíricas sobre la hipótesis de que las decisiones del gobierno central en materia de transferencias a dichos gobiernos responden, al menos parcialmente, al oportunismo político. Sobre la base de estudios similares relativos a otros países se examina la distribución territorial de los "recursos ordinarios", que representan más del 70\% de las transferencias totales dirigidas a las regiones. Dado que estos fondos se asignan discrecionalmente, el gobierno nacional tiene una considerable libertad para entregar transferencias con el fin de obtener réditos políticos.

El tema en cuestión tiene un doble trasfondo teórico. Por una parte, en el marco de diversas recomendaciones normativas de política, las transferencias intergubernamentales se conciben como una manera de corregir la posible mala asignación de recursos causada por externalidades entre jurisdicciones, responder a la necesidad de establecer estándares nacionales sobre los bienes públicos, alcanzar metas interjurisdiccionales de equidad y varios otros objetivos de bienestar a nivel nacional. Por otra parte, el enfoque de economía política resalta la posible desviación respecto de las metas de bienestar nacional debido al uso de las transferencias fiscales por parte del gobierno central como instrumento de clientelismo político. No obstante, la literatura está dividida en cuanto a la estrategia más utilizada por los gobiernos al asignar transferencias. Las dos posturas más comunes son el enfoque del "votante bisagra" (Lindbeck y Weibull, 1993) y la hipótesis de las regiones alineadas (Cox y McCubbins, 1986).

En este trabajo se plantea que el Perú ofrece un terreno fértil para que funcione la llamada maquinaria política. Como en otros países de América Latina, el escenario político es difícil de predecir a lo largo del tiempo y los mecanismos para el control social del comportamiento del gobierno son relativamente débiles, lo que facilita la obtención de rentas más cuantiosas. Además, la clara disociación en el Perú entre los partidos políticos nacionales y los regionales dificulta que los dos niveles de gobierno compartan una estrategia mutuamente beneficiosa. Esto tiene dos consecuencias. En primer lugar, es probable que los objetivos de corto plazo tengan mayor peso al decidir qué regiones y cuáles entidades recibirán transferencias discrecionales. Esta situación promueve el otorgamiento de transferencias más considerables a regiones con mayor riesgo y más rentables políticamente, en las que el electorado es muy volátil. En segundo lugar, la estrategia predominante del gobierno nacional reduce al mínimo el peligro potencial de dar apoyo a dirigentes regionales emergentes, con lo que maximiza la obtención de votos de electorados regionales mayoritariamente opositores. En el marco de este debate, en el presente documento se plantea un modelo empírico con el que se intenta explicar la estrategia de distribución de transferencias del gobierno nacional, modelo que es estimado mediante un panel de datos que abarca el período comprendido entre 2004 y 2010, a objeto de identificar los principales factores de la distribución regional de "recursos ordinarios". Si bien en el modelo empírico se tiene en cuenta un conjunto de consideraciones normativas, los resultados en general coinciden con las hipótesis antes mencionadas.

El resto del documento está organizado de la siguiente manera: en la sección II se analiza la literatura teórica y empírica. En la sección III se describen las instituciones políticas y el mecanismo de financiamiento regional existente en el Perú. El modelo empírico, el enfoque de estimación utilizado y la descripción de los datos se presentan en la sección IV. Los resultados econométricos se muestran en la sección V y en la sección VI se detallan las conclusiones. 


\section{II}

\section{Las teorías y la evidencia empírica existente}

En la explicación que ofrece la economía del sector público acerca del comportamiento de las transferencias fiscales intergubernamentales se distinguen claramente dos enfoques. Uno es puramente normativo y en él se subraya el hecho de que el nivel de gobierno donante tiene como objetivo alguna función de utilidad social de alcance nacional (Musgrave, 1958). Así como los típicos fallos del mercado impiden una asignación eficiente de los recursos en el caso de los bienes privados, los gobiernos subnacionales también pueden tomar decisiones subóptimas sobre el tipo y la cantidad de los bienes públicos específicos que van a proveer. Las externalidades interjurisdiccionales generan una brecha entre los beneficios (o los costos) marginales de orden nacional y aquellos puramente jurisdiccionales (Oates, 1972). La necesidad de establecer estándares nacionales, la existencia implícita (o explícita) de una relación principal-agente entre el gobierno central y los gobiernos subnacionales, el objetivo de tener una distribución más equitativa de los recursos públicos en el territorio nacional y el logro de una eficiente asignación de recursos en el país son justificaciones normativas para las transferencias fiscales (Buchanan, 1950; Inman, 1988; King, 1991; Letelier, 2012). La segunda explicación se basa en el enfoque de la elección pública, el que supone que las decisiones fiscales a nivel nacional se determinarán por las preferencias del llamado "votante mediano". Esto implica que toda asignación de transferencias tiene que seguir las demandas y la distribución geográfica del votante mediano (Boex y Martínez-Vázquez, 2010).

Las transferencias fiscales también pueden analizarse desde la perspectiva de la economía política. En una serie de estudios relativos a la experiencia del New Deal en los Estados Unidos de América, en virtud de la cual se asignaron transferencias considerables a regiones castigadas por la Gran Depresión, se pone en duda que aquellas se hayan destinado realmente a las jurisdicciones que las necesitaban (Arrington, 1970; Reading, 1973). Wright (1974) encontró una correlación sistemática entre el gasto federal per cápita y los votos estatales durante ese período. Similares resultados obtuvieron Inman (1988) y Couch y Shughart (1998), entre otros. En general, en estos estudios se muestra que los argumentos normativos para justificar las transferencias federales a los estados son insuficientes para explicar su comportamiento global, lo que sugiere que las razones políticas jugaron un papel significativo. Este comportamiento se conoce como "clientelismo político" y aumenta el potencial de una asignación inadecuada de fondos públicos cuando el gobierno otorga transferencias fiscales en forma discrecional ${ }^{1}$.

$\mathrm{Si}$ se asume que las transferencias nacionales a las regiones responden, al menos parcialmente, a consideraciones de economía política y que las preferencias de los votantes se basan en motivos personales, el tema central del debate se relaciona con el tipo de votantes regionales que tienen más posibilidades de recibir una mayor cantidad de transferencias fiscales. En la literatura se presentan dos visiones alternativas (Cox, 2010). La primera se conoce como la hipótesis del "votante bisagra" o "votante indeciso", y en ella se establece que las regiones donde abunda este tipo de electores resultan más atractivas políticamente (Lindbeck y Weibull, 1987 y 1993). Case (2001) encontró pruebas para esta hipótesis en el marco de las transferencias federales a los gobiernos locales de Albania. Milligan y Smart (2005) proveen evidencia similar en el caso de las transferencias federales a las cinco provincias más pobres del Canadá. Castells y Solé-Ollé (2005) concluyen que en España los votantes indecisos son importantes en la asignación de transferencias del gobierno central a los gobiernos regionales para obras de infraestructura. Otros casos similares fueron presentados por Wallis (1998) y Gamkhar y Ali (2007) para los Estados Unidos de América, Dahlberg y Johansson (2002) y Johansson (2003) para Suecia, y Gonçalves (2010) para Portugal. Con respecto al Perú, Schady (2000) muestra la presencia de débiles (aunque consistentes) indicios en favor de la hipótesis del votante bisagra en un estudio sobre los recursos del Fondo Nacional de Compensación y Desarrollo Social (FONCODES), los que fueron asignados discrecionalmente a las provincias a comienzos de la década de los años noventa.

\footnotetext{
${ }^{1}$ Como bien señala Grossman (1994), las transferencias basadas en fórmulas también pueden tener un motivo político, dado que la propia fórmula es definida por el gobierno nacional. No obstante, aunque es posible que esto ocurra durante largos períodos, si la fórmula puede modificarse es más probable que las asignaciones puramente discrecionales respondan a una política clientelista.
} 
En la segunda visión se sostiene que las transferencias serán asignadas principalmente a las regiones donde el gobierno nacional tiene más apoyo. Esta es la hipótesis de las "regiones alineadas", con la cual se sugiere que en el marco de la economía política del problema no hay una razón clara que permita explicar por qué el gobierno central debiese ser igualmente generoso con las regiones alineadas y las no alineadas. Cox y McCubbins (1986) afirman que las promesas de los candidatos a sus votantes se hacen en el contexto del clásico equilibrio entre los resultados políticos y el riesgo. Si los políticos prefieren evitar el riesgo (y probablemente así sea) y los votantes indecisos son más riesgosos que los alineados en términos de rédito político, es más probable que las transferencias se entreguen a estos últimos. Solé-Ollé y Sorribas (2008) brindan pruebas respecto de esta teoría en el caso de los municipios españoles. En los Estados Unidos de América las transferencias federales a los estados (Grossman, 1994) y a los gobiernos locales (Ansolabehere y Snyder, 2006) también parecen favorecer esta hipótesis. Resultados similares obtuvieron Biswas, Marjit y Marimoutou (2010) al estudiar las transferencias federales a los estados de la India. Curiosamente, Hanes (2007) considera que en Suecia solo las coaliciones socialistas del gobierno central (en contraposición a las conservadoras) parecen ser más susceptibles a la compra de votos en los municipios con alto porcentaje de votantes partidarios. Gonçalves (2010) concluye que los fondos europeos destinados a los gobiernos locales de Portugal están sesgados hacia los municipios donde la coalición política local gobernante recibe un apoyo más firme. En lo que concierne al Perú, Schady (2000) afirma que se otorgaron más recursos a las provincias alineadas y a aquellas en que el apoyo político al gobierno descendió con respecto a lo registrado en las últimas elecciones presidenciales. En oposición a la hipótesis de las regiones alineadas, Segura-Ubiergo (2007) y Graham y Kane (1998) muestran que durante el mandato presidencial de Fujimori (1990-2001) las transferencias a las regiones se dirigieron discrecionalmente a las zonas que habían prestado el menor apoyo al gobierno durante el referéndum de 1993.

Se han hecho algunos esfuerzos para cerrar la brecha entre ambos enfoques. Dixit y Londregan (1996) señalan que si los partidos son igualmente capaces de recaudar impuestos en todos los grupos, pero más eficientes en beneficiar a sus partidarios, el oportunismo político dominará y se canalizará más dinero a los grupos que apoyen a la coalición gobernante. Las regiones con votantes indecisos tendrán mayores ventajas si los partidos son eficientes en beneficiar a todos los grupos por igual, de modo que la forma de maximizar los votos sea consistente con el enfoque dirigido a los grupos moderados con escaso compromiso ideológico. En un estudio de seguimiento, Dixit y Londregan (1998) muestran que en un mundo de votantes preocupados por la equidad, los grupos de clase media se hacen más atractivos políticamente, dada su mayor concentración de votantes indecisos. En otra rama de la investigación teórica se utiliza el enfoque tradicional de análisis de cartera para examinar la asignación de dinero a las regiones por parte de los políticos, quienes maximizarían las rentas provenientes de varios activos sujetos a diversos grados de riesgo (Díaz-Cayeros, Estévez y Magaloni, 2008; Díaz-Cayeros, 2008).

En otra vertiente de la literatura se destaca el efecto potencial de los grupos de interés en lograr que el gobierno nacional sea más sensible a sus demandas (Olson, 1965). Si bien la dimensión redistributiva está presente en casi todos los aspectos del proceso político, la selección de grupos beneficiarios específicos puede interpretarse como una redistribución táctica (o clientelista) e incluso como un intento de comprar los votos de aquellos que estén más dispuestos a venderlos (Anderson y Tollison, 1988). El sustento a esta hipótesis, en el contexto de la asignación de transferencias por parte del gobierno nacional, ha sido desarrollado por Grossman (1994) y Gamkhar y Ali (2007) en el caso de los Estados Unidos de América; por Porto y Sanguinetti (2001) en Argentina, y por Biswas, Marjit y Marimoutou (2010) en el caso de la India.

Las consecuencias dinámicas de la política clientelista también pueden ser relevantes. En la teoría del ciclo político (Rogoff y Sibert, 1988; Rogoff, 1990) se sugiere que la magnitud de las transferencias fiscales utilizadas como dispositivo táctico se ve potenciada en los años previos a una elección nacional a objeto de elevar las posibilidades de reelección. En numerosos estudios se apoya la existencia de este ciclo (por ejemplo, en Drazen, 2000). El caso peruano, en particular, parece ajustarse a este patrón. Schady (2000) observó que los gastos con cargo al FONCODES aumentaron considerablemente antes de las elecciones. A su vez, Carranza, Chávez y Valderrama (2007) analizaron el período comprendido entre 1970 y 1995 en el país, y concluyeron que la combinación de un fuerte presidencialismo con un escenario político fragmentado ha favorecido el uso de los gastos del gobierno como herramienta para la reelección. 


\section{III}

\section{Las instituciones políticas y el financiamiento regional en el Perú}

\section{El mapa político del Perú}

El Perú tiene un sistema político multipartidario en que se suelen formar alianzas para aumentar las posibilidades electorales. Uno de los partidos más importantes durante el período de la muestra (2004-2010) fue la Alianza Popular Revolucionaria Americana (APRA), que promueve una ideología socialdemócrata con un discurso en favor de la descentralización. A diferencia de otras fuerzas políticas, el APRA es un partido bien organizado y el único partido nacional con fuertes raíces a nivel regional y municipal. Otros tres partidos también fueron importantes en este período: Perú Posible, de centro-izquierda, fundado en 1994 por Alejandro Toledo; el Partido Popular Cristiano, de centro-derecha, que se remonta a los años sesenta del siglo pasado (y tiene alguna representación en el Congreso, pero nunca ha ganado una elección presidencial), y la Alianza por el Futuro, que se formó a partir de la fusión de dos partidos fujimoristas (Cambio 90 y Nueva Mayoría). Con posterioridad al período bajo análisis, el pequeño Partido Nacionalista Peruano se convirtió en una fuerza poderosa al ganar la elección presidencial de 2011.

El proceso de descentralización del Perú recibió un gran impulso en 1989, cuando Alan García — presidente aprista — creó 12 regiones con presidentes regionales. Sin embargo, este importante paso fue anulado temporalmente por el sucesor de García, Alberto Fujimori, quien llevó a cabo un golpe militar en 1992 con el que suspendió la Constitución, cerró el Congreso de la República y obstaculizó la autonomía política regional. En 1993 se aprobó una nueva Constitución, mediante la cual los anteriores gobiernos regionales fueron suplantados por ramas administrativas de la presidencia llamados Consejos Transitorios de Administración Regional (Bensa, 2002). Tras la caída de Fujimori en 2000, el Congreso eligió a un nuevo gobierno de transición que llamó a elecciones presidenciales y parlamentarias. El nuevo Congreso estaría integrado por representantes del nivel regional.

Fujimori fue sucedido por Alejandro Toledo, un candidato de Perú Posible que llegó al poder en 2001 después de una elección muy reñida. En un intento de disminuir el liderazgo del APRA, Toledo lanzó una reforma constitucional en abril de 2002 en pro de la descentralización. Las elecciones presidenciales se celebraron en noviembre de ese año y en ellas se designaron 24 gobiernos regionales (Tanaka, 2002). Si bien ningún candidato de Perú Posible fue elegido, llegaron al poder 12 gobiernos regionales apoyados por el APRA. En julio de 2006, Alan García fue elegido presidente por segunda vez y en noviembre de ese año se celebraron elecciones regionales. El APRA perdió 12 gobiernos regionales en esa elección y terminó con apenas 2 representantes. Como es lógico, las transferencias a las regiones que el gobierno de García había aprobado en el presupuesto fueron reducidas drásticamente entre 2006 y 2007.

La representación política a nivel regional se basa en el nombramiento simultáneo de un presidente, un vicepresidente y un consejo regionales para un período de cuatro años. En los municipios se mantiene un sistema similar. El Perú está dividido en 25 gobiernos regionales, incluida la Provincia Constitucional del Callao (que tiene rango de región) y 1.838 gobiernos municipales. Doce regiones fueron opositoras al gobierno nacional entre 2003 y $2006^{2}$. Solo dos de ellas mantuvieron el mismo gobierno regional y el mismo partido político gobernante después de las elecciones nacionales de 2006. Esto demuestra la alta volatilidad del electorado en estas regiones, lo que proporciona información relevante sobre la ubicación de los votantes indecisos.

\section{El financiamiento regional}

Con la excepción de pequeños retornos por concepto de servicios brindados a nivel regional, las regiones del Perú tienen muy pocos ingresos propios (Vega, 2008). La mayoría de estos provienen de dos de las principales transferencias fiscales del gobierno nacional: los recursos determinados y los recursos ordinarios. Los primeros son alimentados por los impuestos a la industria extractiva, en particular la minería. Una pequeña proporción de los impuestos a las importaciones se asigna también al financiamiento regional, pero solo el

\footnotetext{
2 Es decir, otorgaban menos de un 50\% de apoyo al gobierno nacional.
} 
Callao recibe estos recursos. Una característica distintiva de los recursos determinados es que se asignan de acuerdo con una fórmula específica que solo beneficia a las regiones que producen recursos naturales. Dado que estos se distribuyen de manera muy irregular en todo el territorio, este sistema de participación en los impuestos se traduce en una distribución regional muy desigual. Alrededor del $80 \%$ de los ingresos estatales provenientes de la minería son absorbidos por un tercio de las regiones (Vega, 2008). Además, los gobiernos regionales no reciben todas las transferencias de esta categoría, sino que las comparten con las municipalidades y las universidades locales.

A diferencia de los recursos determinados, los recursos ordinarios se asignan en forma discrecional. Provienen de los ingresos fiscales nacionales generales y no tienen conexión con ninguna entidad pública determinada. Al comienzo del proceso de descentralización, los gobiernos regionales se hicieron cargo automáticamente de una proporción mucho mayor de las responsabilidades de gasto público nacional. Por este motivo, en 2003 el Ministerio de Economía y Finanzas introdujo las transferencias regionales como una nueva partida presupuestaria, aunque los recursos ordinarios no están incluidos oficialmente en el marco institucional de la financiación de la descentralización del país. Una posible interpretación de la Ley de Descentralización Fiscal es que los recursos ordinarios deben ser concebidos como un instrumento transitorio para apoyar a las regiones hasta que algunos impuestos específicos queden legalmente bajo su control. Hasta ahora no hay ninguna norma específica que impida la asignación discrecional de recursos ordinarios a las regiones, las que a su vez los utilizan para financiar tanto sus gastos de personal como las inversiones locales. En los últimos años, el aumento de la proporción de las inversiones regionales se financia con los recursos determinados, y los recursos ordinarios se emplean principalmente para cubrir gastos corrientes.

El total de las transferencias a los gobiernos subnacionales (incluidos los municipios) como proporción del presupuesto del gobierno general aumentó del $27 \%$ en 2005 al 37\% en 2011 (véase el cuadro 1). Este notable incremento se debe a tres fenómenos importantes. En primer lugar, se delegaron algunas nuevas funciones a las regiones durante el período analizado. En segundo lugar, las transferencias no discrecionales se expandieron considerablemente a raíz de un alza significativa del precio del oro, el cobre y el petróleo en el período de estudio. En tercer lugar, el sólido y persistente crecimiento económico peruano de ese período contribuyó a elevar el aumento de las transferencias. A pesar de que el Ministerio de Economía y Finanzas está involucrado en la toma de decisiones sobre la distribución de las transferencias fiscales a los gobiernos regionales, gran parte de los recursos ordinarios provienen del presupuesto del gobierno nacional. Este hecho, sumado a la falta de financiación de las regiones, ha tendido a reforzar el uso de las negociaciones políticas como un factor importante a la hora de distribuir estos recursos.

Perú: transferencias a los gobiernos subnacionales, 2005-2011

\begin{tabular}{|c|c|c|c|c|c|c|c|}
\hline Tipo de transferencia & 2005 & 2006 & 2007 & 2008 & 2009 & 2010 & 2011 \\
\hline Presupuesto total del gobierno general (en miles de millones de dólares) & 17,64 & 19,45 & 22,51 & 27,69 & 27,70 & 31,76 & 33,63 \\
\hline Transferencias a todos los gobiernos subnacionales (en miles de millones de dólares) & 4,80 & 5,47 & 6,79 & 9,97 & 11,03 & 11,48 & 12,30 \\
\hline Transferencias como porcentaje del presupuesto del gobierno general & 27,00 & 28,00 & 30,00 & 36,00 & 40,00 & 36,00 & 37,00 \\
\hline Total de las transferencias a los gobiernos regionales (en miles de millones de dólares) & 1,67 & 2,04 & 2,94 & 4,54 & 4,97 & 5,58 & 5,35 \\
\hline Recursos ordinarios (en miles de millones de dólares) & 1,46 & 1,67 & 2,17 & 3,41 & 3,66 & 4,06 & 4,03 \\
\hline Recursos ordinarios como porcentaje de las transferencias regionales & 87,00 & 82,00 & 74,00 & 75,00 & 74,00 & 73,00 & 75,00 \\
\hline Recursos determinados (en miles de millones de dólares) & 0,21 & 0,37 & 0,77 & 1,13 & 1,31 & 1,52 & 1,32 \\
\hline Recursos determinados como porcentaje de las transferencias regionales & 13,00 & 18,00 & 26,00 & 25,00 & 26,00 & 27,00 & 25,00 \\
\hline
\end{tabular}

Fuente: elaboración propia sobre la base de la Ley de Presupuesto General de la República del Perú. 


\section{IV}

\section{Pruebas de la hipótesis}

\section{El modelo empírico}

En el marco de la hipótesis de este trabajo, el comportamiento de los mandatarios nacionales en cuanto a la asignación de transferencias fiscales discrecionales (grants) está generalmente determinado por una combinación de factores sociodemográficos (SDEM), económicos $(E C O N)$ y políticos $(P O L)$. Se asume que el potencial de sesgo oportunista del gobierno está restringido por diversos factores. Primero, existe un marco institucional que limita la posibilidad de que las transferencias se utilicen con fines electorales. Segundo, todas las coaliciones políticas responden, al menos parcialmente, a las preferencias del votante mediano. Tercero, en general la ideología también importa. De este modo, el modelo de panel puede ser expresado por la ecuación (1), donde $r$ representa la región y $t$ el tiempo:

$$
\begin{gathered}
\text { grant }_{r t}=\alpha+\beta_{1} \times P O L_{r t}+\beta_{2} \times E C O N_{r t}+ \\
\beta_{3} \times S D E M_{r t}+\mu_{r t}+\varepsilon_{r t}
\end{gathered}
$$

Una variable clave para discriminar entre la hipótesis del votante indeciso y la de las regiones alineadas es el grado de apoyo político regional al gobierno nacional que está en el poder. Como en la mayoría de los estudios empíricos sobre este tema, se exploran aquí dos variables relacionadas. La primera es la participación de cada circunscripción regional que votó a favor del actual gobierno nacional (ng.votes). Un efecto positivo indica que se está favoreciendo a las regiones alineadas. En segundo lugar, en algunos estudios se indica que las regiones con votantes indecisos son aquellas con un apoyo cercano al $51 \%$ de los electores en favor del gobierno nacional. Este porcentaje se denomina votos marginales (mar.votes). Ambas variables son controladas por la población electoral de la región.

Se pueden esperar los siguientes resultados: i) si

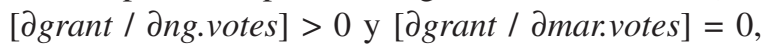
se supone que los votantes alineados predominan a la hora de decidir el otorgamiento de transferencias;

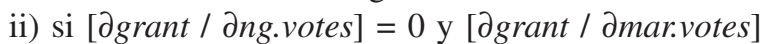
$<0$, se deduce que se está favoreciendo a las regiones con votantes indecisos, y iii) si [jgrant / Jng.votes $]<0$ yдวgrant / Jmar.votes $] \geq 0$, la respuesta depende de la composición de los votantes de la región. Un electorado volátil en las regiones con bajos ng.votes (que son las más favorecidas en este caso) indica que se tiene como objetivo a los electores indecisos. Incluso si este no fuera el caso, la opción iii) es compatible con que el gobierno nacional tenga una habilidad especial para llegar a los beneficiarios de las regiones con bajos ng.votes y una capacidad similar para aplicar impuestos en todas las regiones (Dixit y Londregan, 1996). En el Perú, la situación iii) es la más probable, ya que en un escenario político de rápida evolución, los votantes generalmente no tienen la visión de futuro necesaria para castigar al gobierno si ello se traduce en una reducción de las transferencias a su región. En este sentido, es posible que el ciclo político tradicional, tal como lo definen Rogoff y Sibert (1988), se vea alterado por la oportunidad de retirar los beneficios de quienes apoyan al gobierno nacional para dárselos a los oponentes inmediatamente después de que dicho gobierno resulte electo.

Otro punto a considerar es si el grado de apoyo de los gobiernos regionales es relevante desde el punto de vista de la estrategia del gobierno nacional para mantenerse en el poder. Considérese la hipótesis de que la lealtad política a los partidos regionales (rg.votes) representa una amenaza para el gobierno nacional cuando los dirigentes regionales ganan popularidad. Por ejemplo, Dickovick (2006) sostiene que la descentralización municipal en el Perú y otros países de América Latina estaba implícitamente dirigida a debilitar el nivel intermedio del gobierno. Así como se espera que los gobiernos regionales con alto grado de apoyo reciban menos transferencias, también se espera que los gobiernos regionales que pertenecen al mismo partido del presidente nacional tengan beneficios especiales. Se incluye para este caso una variable muda (D.REGNAT).

Entre las variables políticas, se supone que las autoridades nacionales son sensibles al cabildeo y los grupos de presión, no obstante que este canal de demandas locales no resulta fácil de observar. Se puede distinguir entre el cabildeo (lobby) — que se expresa en la forma de individuos, organizados o no, que intentan presentar sus demandas-y otras formas más radicales de presionar al gobierno nacional, las que suelen dar como resultado un evidente conflicto político a nivel regional (conflicts). Ambas formas de presión pueden convertirse en una amenaza para la capacidad del 
gobierno nacional de mantenerse en el poder, de modo que las transferencias posiblemente sean sensibles a ellas. Además, las regresiones incluyen efectos fijos temporales que capturan elementos del ciclo político, así como una variable muda para detectar el efecto de las regiones en las que el gobierno nacional haya obtenido más de un $50 \%$ de votos en las últimas elecciones presidenciales $(D .50)$.

Con respecto a las razones no oportunistas para asignar transferencias, se espera que el costo y la demanda afecten a la voluntad del gobierno de apoyar a las regiones más urbanizadas o más densamente pobladas (urban y dens). Del mismo modo, la capacidad potencial de las transferencias para compensar a las regiones pobres explica la influencia que puede ejercer la tasa de pobreza (pov). Una distribución justa de los ingresos fiscales exige más transferencias a las regiones en las que los impuestos per cápita son menores. Por lo tanto, si los impuestos per cápita recaudados por el nivel nacional en la región (tax) y los recursos determinados (canon) son elevados, probablemente disminuya el apoyo financiero por parte del gobierno nacional.

\section{La estrategia de estimación}

En la estrategia de estimación se consideran dos especificaciones econométricas del modelo general. En la primera se utilizan las transferencias per cápita como variable endógena, tal como se efectúa en estudios similares. Podría decirse que las desviaciones de una asignación per cápita equivalente responden a los factores mencionados (véase la ecuación (1)). Países como el Canadá y España son categóricos en la igualación de la capacidad fiscal per cápita entre las regiones, lo que se expresa en una metodología de cálculo de transferencias que contiene consideraciones explícitas respecto del costo local de los bienes públicos en cada región, de las metas de nivelación fiscal, de algunas mediciones de las necesidades locales, de la base imponible jurisdiccional y de varios otros indicadores (Dahlby, 2008; Bosch, 2009).

La segunda especificación se centra en el nivel de las transferencias fiscales asignadas (en letras mayúsculas). Todas las variables se presentan en logaritmos naturales (ln). A diferencia del modelo per cápita, en este enfoque se asume que la asignación de cada año se basa en el monto otorgado en el año anterior. Un desafío evidente en el uso de este modelo es la posible correlación entre el grado de retardo de la variable dependiente, $\ln ($ GRANT_1), y el término de error. La estimación simple de este modelo por mínimos cuadrados ordinarios da como resultado estimaciones de parámetros sesgadas e inconsistentes
(Greene, 2003). Para hacer frente a esto, se utiliza una estimación mediante el método generalizado de momentos (MGM) que, bajo supuestos razonables, da como resultado estimadores consistentes, asintóticamente normales y asintóticamente eficientes (Hansen, 1982). El resto de las variables (no endógenas) y los efectos fijos de las regiones son utilizados como instrumentos en el modelo referido.

\section{Los datos}

En el anexo se presenta una lista completa de las variables, con sus definiciones y fuentes. GRANT representa las transferencias discrecionales del gobierno central a los gobiernos regionales $(O R)$. Estas son asignadas anualmente por el Ministerio de Economía y Finanzas. Los datos sobre esta variable se tomaron del presupuesto anual correspondiente, que se publica en el Portal de Transparencia Económica del ministerio del ramo. El debate político sobre el presupuesto se basa en una propuesta del gobierno nacional, que se convierte en ley cuando la aprueba el Congreso de la República. Todos los datos se expresan en nuevos soles constantes de 2006. La misma fuente se utilizó para la variable canon, que registra todas las transferencias de los recursos determinados. Las partidas presupuestarias de esta categoría no estaban disponibles antes de 2007, por lo tanto, para el período 2004-2006 la variable se construyó mediante la suma de todos los ingresos (canon) provenientes de la explotación de recursos naturales de cada región. La variable tax procede de la Superintendencia Nacional de Administración Tributaria, que es la división administrativa encargada de la recaudación de impuestos. Oficialmente, estos datos son recopilados a nivel regional sobre la base de la dirección fiscal de los contribuyentes.

Con respecto a las variables políticas, conflicts representa el número de iniciativas privadas de origen regional destinadas a presionar al gobierno para que aumente la financiación de áreas específicas. Esta variable se elaboró a partir de información proporcionada por la Defensoría del Pueblo, que clasifica estos conflictos por regiones. Las fuentes más comunes de cabildeo (lobby) incluyen a autoridades locales (municipales) y regionales, y a representantes sindicales de funcionarios públicos, en particular de los docentes. Las variables electorales se obtuvieron del Jurado Nacional de Elecciones, que gestiona los procesos electorales y registra sus resultados.

Las variables sociodemográficas fueron proporcionadas por el Instituto Nacional de Estadística e Informática (INEI). Esta entidad recoge dos series de datos básicos: el Censo Nacional de Población y Vivienda, cuya última versión corresponde a 2007, y la Encuesta Nacional de 
Hogares, que se lleva a cabo anualmente. Desde 1997, el INEI publica año a año un informe sobre la tasa de pobreza basado en dicha encuesta.

Por último, cabe hacer algunos alcances sobre los datos. Primero, a pesar de haber optado por utilizar los recursos ordinarios establecidos en el presupuesto, es probable que en las cifras se sobrestimen los gastos reales de las regiones. Sin embargo, se supone que el presupuesto en sí mismo recoge las presiones políticas con bastante exactitud. Segundo, los datos utilizados para la variable lobby están potencialmente sesgados a la baja, dado que algunos episodios relacionados con el cabildeo pueden pasar desapercibidos si no se comunican a las autoridades correspondientes mediante una nota oficial. Lo mismo puede decirse de la variable conflicts. Tercero, dado que algunas de las series relacionadas que se incluyen solo están disponibles para períodos anteriores al período de estudio, las regresiones reportadas cubren solamente hasta 2010.

\section{V}

\section{Resultados econométricos}

\section{Variables económicas}

Puede esperarse que las transferencias se vean afectadas negativamente por las variables tax y canon en la regresión per cápita (véase el cuadro 2). Aunque esto es precisamente lo que ocurre, solo canon parece ser significativa. Una posible explicación es que las transferencias fiscales con cargo a los recursos ordinarios se utilicen como una herramienta de compensación, haciendo que las regiones con pocos recursos naturales reciban más transferencias. El signo negativo - aunque no significativo- del coeficiente estimado para tax es probable que refleje una función similar de los recursos ordinarios con respecto a la base imponible general de las regiones. No obstante, este último efecto compensatorio parece ser más débil. Las regresiones en niveles proporcionan resultados ligeramente diferentes (véase el cuadro 3). Una diferencia importante es que $\ln$ (CANON) parece tener un efecto positivo en el nivel de las transferencias fiscales. Esto puede reflejar el hecho de que las regiones que reciben elevadas transferencias de recursos determinados (es decir, que tienen un valor elevado de CANON) también manifiestan una mayor necesidad de infraestructura pública. Dado que las regiones son responsables de esta área de gobierno, deberían recibir más recursos para su gestión. Sin embargo, la demanda de infraestructura no se relaciona necesariamente con el valor del canon per cápita, lo que explica que los signos de los coeficientes estimados en los cuadros 2 y 3 varíen entre sí. A diferencia de las transferencias per cápita, el nivel de los impuestos tiene un importante efecto negativo en el nivel de las transferencias fiscales. Esto demuestra que, independientemente de la población, las regiones con una mayor base imponible reciben menos transferencias.

\section{Variables sociodemográficas}

Las estimaciones presentadas muestran que pov no es significativa en ninguna de las regresiones, excepto en MGM3 (véase el cuadro 3). Esto es compatible con la asignación oficial de las funciones regionales definidas en la reforma constitucional orientada a la descentralización (ley 27.680), la Ley de Bases de la Descentralización (ley 27.783) y la Ley Orgánica de Gobiernos Regionales (ley 27.867), aprobadas en 2002. Se pretende que las regiones promuevan el desarrollo económico regional, las inversiones regionales y todos los servicios y actividades de su competencia. Aunque se les asignan numerosas tareas, no se menciona explícitamente que las regiones sean responsables de los programas de mitigación de la pobreza, lo que ha de tenerse en cuenta al interpretar los resultados anteriores. Si bien se comparte la responsabilidad de la provisión de educación básica, servicios de salud y promoción del empleo, el gobierno nacional gestiona tanto el financiamiento como la administración de los programas sociales.

Con respecto a la densidad (density), el coeficiente positivo de las estimaciones per cápita sugiere que las regiones con mayor densidad de población probablemente tengan mayores externalidades interjurisdiccionales y costos de congestión. Esto eleva el costo per cápita de los bienes públicos regionales (véanse las regresiones FE2 y FE3 en el cuadro 2), pero no el nivel de las transferencias (Litvack y Oates, 1971), y explica además el signo de urban. Esta variable registra el grado de distribución desigual de la población nacional entre las regiones. Como ello exacerba las externalidades y los problemas de congestión, es posible que se asignen más transferencias a las regiones con alto nivel de urbanización. En ambos cuadros se presentan resultados consistentes con este argumento. 
La variable pop captura un efecto directo sobre la demanda en los bienes públicos regionales y, de esta manera, afecta positivamente a las transferencias. Dado que las transferencias se dividen por la población en el cuadro 2 , esta variable se omite de estas regresiones, mientras que se incluye en el cuadro 3. Los resultados muestran que un incremento de la población de un $1 \%$ conduce a un aumento de las transferencias fiscales entre un $0,63 \%$ y un $0,52 \%$ (véanse las regresiones MGM2 y MGM3 en el cuadro 3).

\section{Las variables de la economía política}

En cuanto a las consideraciones de economía política, la variable lobby solo es significativa en las regresiones en niveles. En el caso de la variable conflicts, ocurre exactamente lo contrario. Esto es coherente con la naturaleza de ambas variables. Dado que los conflictos se originan en la presión de grupos bien identificados que representan una amenaza permanente a la estabilidad política nacional, es de esperar que se produzca un incremento permanente de las transferencias per cápita a lo largo del tiempo (véase el cuadro 2). Por otra parte, la variable lobby está construida sobre la base de las demandas expresadas por diversos grupos y personas a los representantes regionales. Como su origen es menos específico, esta variable no plantea una amenaza visible para las autoridades nacionales. Esto también concuerda con el hecho de que la variable lobby sea significativa solo en las regresiones en niveles, lo que supone de manera implícita que el aumento del cabildeo no necesariamente se traduce en mayores transferencias por habitante.

Se analizaron varias mediciones del apoyo de los votantes al gobierno nacional y a los gobiernos regionales. Como era de esperar, los resultados muestran que los gobiernos regionales que cuentan con un apoyo elevado (alto valor de rg.votes) reciben menos transferencias per cápita (véase el cuadro 2). Este hecho está en consonancia con el intento del gobierno nacional de amortiguar la potencial competencia política en un contexto relativamente no ideológico. En las regresiones en niveles se observa un resultado similar (véase el cuadro 3). Coincidentes con este resultado, los datos econométricos también sugieren que se espera obtener un mayor número de nuevos votos con el envío de recursos a las regiones no alineadas. Ello se ve refrendado cuando se emplea $D 50+$ en lugar de variables de apoyo al gobierno (véase FE2 en el cuadro 2 y MGM2 en el cuadro 3). Esta variable muda parece ser especialmente significativa para explicar las transferencias per cápita (véase el cuadro 2), en cuyo caso los resultados son los mismos, aun cuando las variables ng.votes, rg.votes y $\mathrm{D} 50+$ se incluyan juntas en la regresión (FE3, FE4 y FE5). Por su parte, las transferencias se encuentran relacionadas positivamente con el número de electores a nivel regional (véase MGM1 en el cuadro 3). Debido a la alta correlación entre la población electoral y la población, estas variables independientes no se utilizan juntas. Una lectura directa de MGM1 indica que un aumento del $1 \%$ en la masa de electores regionales conduce a que las transferencias fiscales crezcan un $0,4 \%$. Se realizó una regresión adicional para incluir a los votantes marginales (marvotes) en FE4 y MGM4. Esto se efectúa conjuntamente con la inclusión de 50+ en la regresión. En ambos casos, la variable mar.votes es significativa y tiene un coeficiente positivo en FE4, lo que va en contra de cierta evidencia en favor de la hipótesis del votante indeciso. Este hecho tiene una doble interpretación. En primer lugar, sugiere que los votantes indecisos no están ubicados en regiones donde el apoyo político al gobierno nacional bordea el 50\%, como señalan algunos estudios empíricos. En segundo lugar, revela que el gobierno nacional asigna el más alto rédito político al otorgamiento de transferencias a las regiones opositoras. Esto puede explicarse por el hecho de que aplicar impuestos a los grupos de apoyo, que en este caso deben definirse como los grupos que reciben menos transferencias, no conduce a una pérdida importante de votos respecto de los votos adicionales obtenidos mediante el apoyo a grupos de la oposición (Dixit y Londregan, 1998). Aunque difícil de mantener en el largo plazo, una estrategia de este tipo puede ser efectiva al comienzo del mandato, como sugiere el valor relativamente mayor del efecto temporal de 2007 (véase el cuadro 2). Al analizar el comportamiento de los votantes de las regiones opositoras en relación con las regiones partidarias del gobierno, puede observarse un patrón de mayor consistencia temporal. De las 12 regiones opositoras ${ }^{3}$ durante el período 2003-2006, solo 2 mantuvieron el mismo gobierno regional y el mismo partido político gobernante después de las elecciones nacionales de 2006. Esto evidencia la alta volatilidad del electorado, lo que sugiere que las regiones opositoras son las más propensas a tener votantes inestables, lo que refrenda la hipótesis del votante indeciso.

También pueden encontrarse pruebas del ciclo político clásico. Con respecto al mandato del presidente Toledo (2004-2006), el primer año de la muestra (2004) registra un valor menor que los restantes efectos temporales, lo que también ocurre en 2009. Es decir, en los años alejados de las elecciones presidenciales se registran coeficientes más bajos y menos significativos (véase el cuadro 2).

\footnotetext{
${ }^{3}$ Es decir, las que otorgaban menos de un $50 \%$ de apoyo al gobierno nacional.
} 


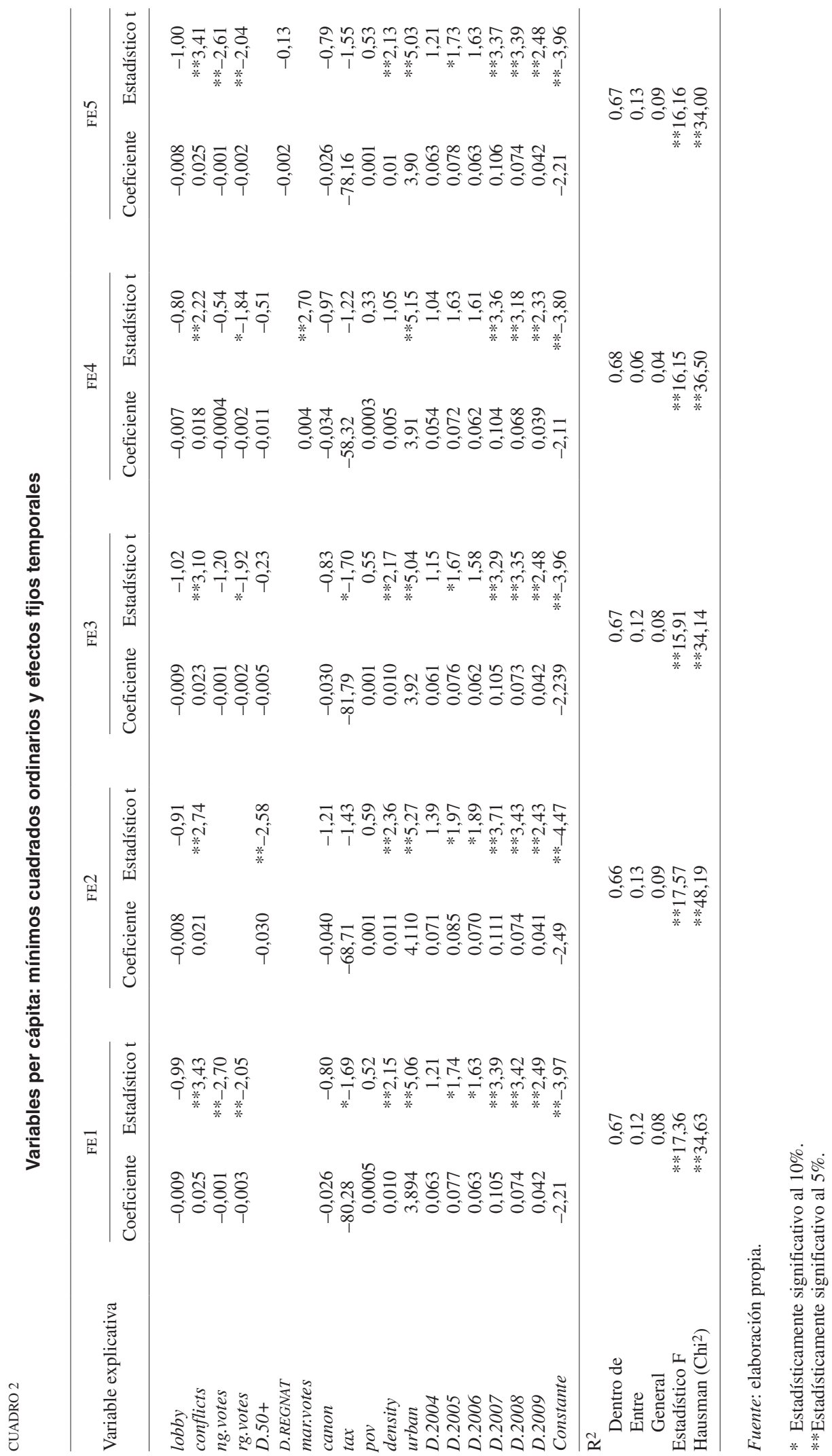




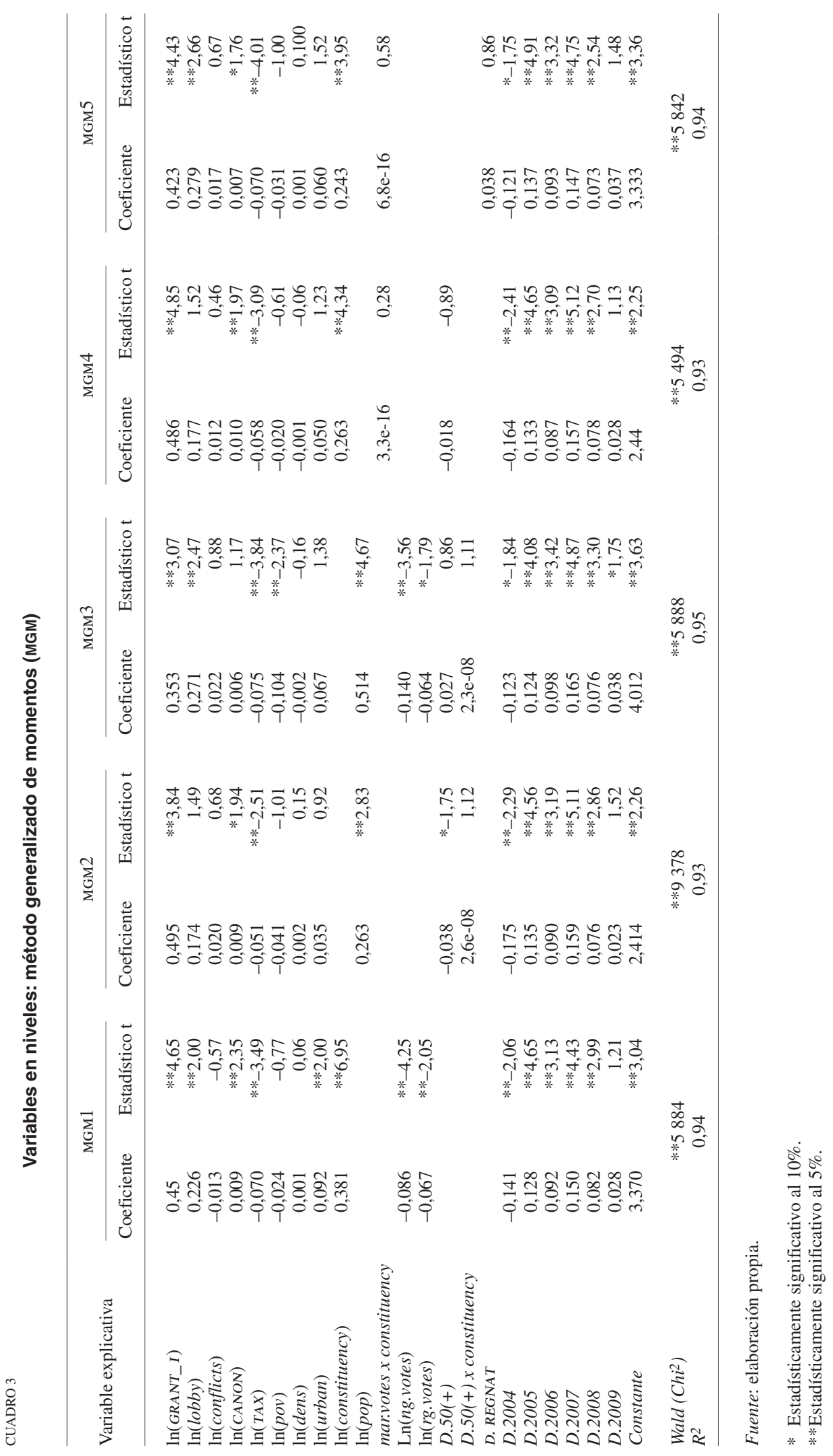




\section{Resumen de las hipótesis frente a la evidencia empírica}

En el cuadro 4 se muestran los principales resultados, con un resumen de las hipótesis probadas y los correspondientes resultados econométricos. Las variables empleadas para detectar el comportamiento oportunista del gobierno nacional son, en general, significativas y presentan en la teoría profundos efectos. En primer lugar, las transferencias fiscales discrecionales parecen ser impulsadas por los electores indecisos. Por una parte, la hipótesis de las regiones alineadas queda claramente rechazada, teniendo en cuenta los coeficientes negativos de ng.votos y $D .50+$, los que indican que las regiones no alineadas reciben el mayor porcentaje de los beneficios. Por otra parte, la composición de los electorados en las regiones opositoras parece ser muy volátil (como ya se indicó), lo que refuerza la afirmación de que en dichas regiones se encuentran los votantes más indecisos de la muestra. Un segundo ingrediente de economía política es que las regiones cuyo electorado apoya fervientemente al presidente en el poder reciben menos transferencias discrecionales. El coeficiente negativo y significativo rg.votes apoya esta afirmación al dar pruebas de que los dirigentes regionales populares pueden ser vistos como una amenaza política para el gobierno nacional. La tercera evidencia es que el cabildeo (lobby) y los grupos de interés (conflicts) también importan... Cuarto, los resultados detectan también la existencia de un ciclo electoral, ya que los años cercanos a las elecciones presidenciales registran efectos temporales mayores y más significativos. Por último, la falta de significación estadística de D.REGNAT, que se emplea como variable proxy del grado de interacción ideológica entre los niveles nacionales y regionales del gobierno, puede ser interpretada como una señal de que la ideología no ocupa un lugar destacado en el criterio de asignación de las transferencias.

Las consideraciones normativas también cumplen un papel importante. Como era de esperar, tanto la población (pop) como la densidad de población (density) y la urbanización (urban) afectan positivamente a las transferencias fiscales. Sin embargo, la pobreza (pov) no parece ser un factor significativo, lo que es coherente con las competencias otorgadas a las regiones en el Perú. Del mismo modo, los ingresos derivados de los impuestos a la minería per cápita (canon) afectan negativamente a las transferencias fiscales por habitante, así como los impuestos aparecen como significativos en la regresiones $\ln ($ GRANT). Como se señaló, el efecto positivo de canon en $\ln$ (GRANT) puede reflejar el hecho de que las regiones que reciben un alto nivel de recursos determinados probablemente requieran mayor inversión pública.

CUADRO 4

Efectos esperados y resultados econométricos

\begin{tabular}{|c|c|c|c|c|}
\hline \multirow{2}{*}{ Variable } & \multicolumn{2}{|c|}{ Efecto sobre grcap } & \multicolumn{2}{|c|}{ Efecto sobre $\ln (G R A N T)$} \\
\hline & Esperado & Estimado & Esperado & Estimado \\
\hline lobby & + & NS & + & + \\
\hline conflicts & + & + & + & NS \\
\hline ng.votes & $?$ & - & $?$ & - \\
\hline rg.votes & - & - & - & - \\
\hline Constituency & & & + & + \\
\hline$D .50+$ & $?$ & - & $?$ & - \\
\hline D. $50+\times$ constituency & & & $?$ & NS \\
\hline marvotes & $?$ & + & $?$ & NS \\
\hline marvotes $\times$ constituency & & & + & NS \\
\hline canon & - & - & $?$ & + \\
\hline $\operatorname{tax}$ & - & NS & $?$ & - \\
\hline pov & $?$ & NS & $?$ & - \\
\hline density & + & + & + & NS \\
\hline рор & + & + & & \\
\hline urban & $?$ & + & $?$ & + \\
\hline D.REGNAT & + & NS & + & NS \\
\hline
\end{tabular}

Fuente: elaboración propia.

grcap: transferencias por habitante. constituency: circunscripción.

NS: no significativo. 
Finalmente, otras hipótesis relevantes pueden someterse a prueba más allá del ámbito específico de este trabajo. Un estudio empírico sobre este tema en el área municipal podría constituir un aporte muy importante. Los gobiernos locales del Perú también reciben recursos ordinarios que se utilizan especialmente para financiar los programas sociales que llevan a cabo los municipios por orden del gobierno central. Aunque son

\section{VI}

\section{Conclusiones}

La presente investigación arroja luz sobre la economía política de la distribución regional de las transferencias fiscales discrecionales en el Perú, denominadas "recursos ordinarios". A diferencia de lo observado en estudios empíricos similares relativos a otros países, aquí se concluye que las regiones en que el gobierno nacional tiene el menor apoyo, en general, reciben la mayor parte de los beneficios. Una posible explicación para este resultado se encuentra en el razonamiento de Dixit y Londregan (1996), en que se asume que el gobierno nacional puede tener una ventaja comparativa al dirigir las transferencias fiscales a los grupos de la oposición, transfiriendo a la vez el costo de esta maniobra política a los grupos que lo apoyan. Es más probable que este escenario tenga lugar al comienzo del período de gobierno, lo que es compatible con los resultados de las regresiones. Sin embargo, la menos cuantiosos que las transferencias de recursos ordinarios regionales, se puede presumir que al menos en cierto grado su asignación esté también motivada políticamente. Otra derivación de este estudio que puede resultar interesante se basa en la forma en que los municipios determinan la recaudación de impuestos sobre el patrimonio; por ejemplo, quiénes deben pagar y qué intensidad tiene la tarea de recaudación. asociación positiva entre las regiones opositoras y su mayor proporción de votantes volátiles sugiere que a largo plazo la hipótesis del votante indeciso resulta válida.

Los resultados también indican que el gobierno nacional es sensible al tamaño del electorado regional $\mathrm{y}$ al peligro de la posible competencia de dirigentes locales poderosos, lo que se refleja en el bajo nivel de las transferencias a los gobiernos regionales políticamente fuertes. Al asignar las transferencias, el gobierno nacional también parece ser sensible a la presión ejercida por grupos privados organizados y a los conflictos regionales. Las consideraciones normativas también son relevantes. Se otorgan más fondos discrecionales a las regiones densamente pobladas y urbanizadas y a aquellas con menor base imponible y menos relacionadas con los recursos mineros. 
ANEXO

CUADRO A1

Definiciones y fuentes de las variables

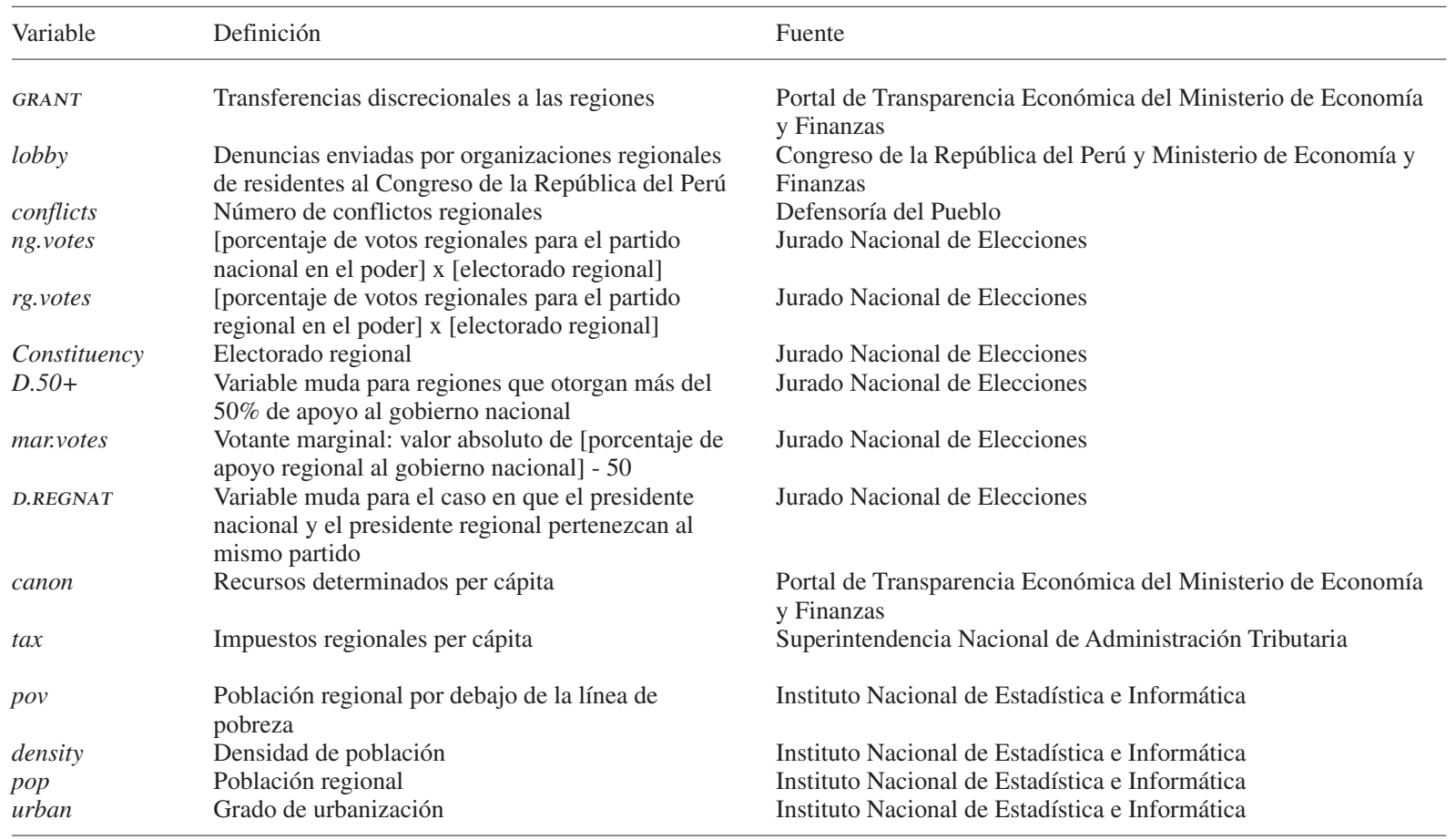

Fuente: elaboración propia.

Anderson, G. y R.D. Tollison (1988), "Democracy, interest groups, and the price of votes", Cato Journal, vol. 8, № 1, Washington, D.C., CATO Institute.

Ansolabehere, S. y J.M. Snyder (2006), "Party control of state government and the distribution of public expenditures", The Scandinavian Journal of Economics, vol. 108, № 4, Wiley.

Arrington, L.J. (1970), "Western agriculture and the new deal", Agricultural History, vol. 44, No 4, Winter Park, Agricultural History Society.

Bensa, J. (2002), "El sistema electoral subnacional en Perú y Chile entre 1980 y 2002", Elecciones, No 1, Lima, Oficina Nacional de Procesos Electorales.

Biswas, R., S. Marjit y V. Marimoutou (2010), "Fiscal federalism, state lobbying and discretionary finance: evidence from India", Economics \& Politics, vol. 22, $\mathrm{N}^{\circ}$ 1, Wiley Blackwell.

Boex, J. y J. Martínez-Vázquez (2010), "The Determinants of the Incidence of Intergovernmental Grants: A Survey of the International Experience", Atlanta, Andrew Young School of Policy Studies, Georgia State University, inédito.

Bosch, N. (2009), "El sistema de nivelación fiscal entre las comunidades autónomas", Revista de Estudios Regionales, Número extraordinario (VIII), Universidades Públicas de Andalucía.

Buchanan, J. (1950), "Federalism and fiscal equity", American Economic Review, vol. 40, № 4, Nashville, Tennessee, American Economic Association.
Carranza, L., J. Chávez y J. Valderrama (2007), "La economía política del proceso presupuestario: El caso peruano", Documento de trabajo, $\mathrm{N}^{\circ} \mathrm{CS}-102$, Washington, D.C., Banco Interamericano de Desarrollo.

Case, A. (2001), "Election goals and income redistribution: recent evidence from Albania", European Economic Review, vol. 45, $\mathrm{N}^{\mathrm{o}} 3$, Amsterdam, Elsevier.

Castells, A. y A. Solé-Ollé (2005), "The regional allocation of infrastructure investment: the role of equity, efficiency and political factors", European Economic Review, vol. 49, № 5, Amsterdam, Elsevier.

Couch, J.F. y W. Shughart II (1998), The Political Economy of the New Deal, Cheltenham, The Independent Institute.

Cox, G. (2010), "Swing voters, core voters and distributive politics", Political Representation, I. Shapiro y otros (eds.), Cambridge, Cambridge University Press.

Cox, G. y M. McCubbins (1986), "Electoral politics as a redistributive game", Journal of Politics, vol. 48, $\mathrm{N}^{\circ}$ 2, Cambridge, Cambridge University Press.

Dahlberg, M. y E. Johansson (2002), "On the vote-purchasing behavior of incumbents governments", American Political Science Review, vol. 96, No 1, Cambridge, Cambridge University Press.

Dahlby, D. (2008), "The Canadian federal-provincial fiscal equalization system", CEsifo DICE Report, vol. 6, № 1, Munich, Universidad de Munich. 
Díaz-Cayeros, A. (2008), "Electoral risk and redistributive politics in Mexico and the United States", Studies in Comparative International development, vol. $43, \mathrm{~N}^{\circ} 2$, Springer.

Díaz-Cayeros, A., F. Estévez y B. Magaloni (2008), "Strategies of vote buying: social transfers, democracy and welfare in Mexico", Stanford, Universidad de Stanford.

Dickovick, J.T. (2006), "Municipalization as central government strategy: central-regional-local politics in Peru, Brazil, and South Africa", Publius: The Journal of Federalism, vol. 37, $\mathrm{N}^{\mathrm{o}} 1$, Oxford University Press.

Dixit, A. y J. Londregan (1998), "Ideology, tactics, and efficiency in redistributive politics", The Quarterly Journal of Economics, vol. 113, No 2, Oxford, Oxford University Press, mayo.

(1996), "The determinants of success of special interests in redistributive politics", The Journal of Politics, vol. 58, № 4, Cambridge, Cambridge University Press.

Drazen, A. (2000), Political Economy in Macroeconomics, Princeton, Princeton University Press.

Gamkhar, S. y H. Ali (2007), "Political economy of grant allocations: the case of federal highway demonstration grants", Publius: The Journal of Federalism, vol. 38, $\mathrm{N}^{\mathrm{o}}$ 1, Oxford University Press.

Gonçalves, L. (2010), "Determinants of the assignments of EU funds to Portuguese municipalities", Public Choice, por aparecer.

Graham, C. y C. Kane (1998), "Opportunistic government or sustaining reform: electoral trends and public expenditure patterns in Peru 1990-1995”, Latin American Research Review, vol. 33, № 1, Pittsburgh, Latin American Studies Association.

Greene, W. (2003), Econometric Analysis, New Jersey, Prentice Hall. Grossman, P. (1994), "A political theory of intergovernmental grants", Public Choice, vol. 78, № 3-4, Springer.

Hanes, N. (2007), "Temporary grant programmes in Sweden and central government behavior", European Journal of Political Economy, vol. 23, № 4, Amsterdam, Elsevier.

Hansen, L.P. (1982), "Large sample properties of generalized method of moments estimators", Econometrica, vol. 50, No 4, Nueva York, The Econometric Society.

Inman, R. (1988), "Federal assistance and local services in the United States: the evolution of a new federalist order", Fiscal Federalism: Quantitative Studies, H. Rosen (ed.), Chicago, University of Chicago Press.

Johansson, E. (2003), "Intergovernmental grants as a tactical instrument: empirical evidence from Swedish municipalities", Journal of Public Economics, vol. 87, № 5-6, Amsterdam, Elsevier.

King, D. (1991), "Grants as source of local government finance", Local Government: An International Perspective, J. Owens y G. Panella (eds.), Amsterdam, North Holland.

Letelier, L. (2012), "Teoría y práctica de la descentralización fiscal", Santiago de Chile, Ediciones Católica de Chile.

Lindbeck, A. y J. Weibull (1993), "A model of political equilibrium in a representative democracy", Journal of Public Economics, vol. 51, Amsterdam, Elsevier.
(1987), "Balanced-budget redistribution as the outcome of political competition", Public Choice, vol. 52, No 3, Springer.

Litvack, J. y W. Oates (1971), "Group size and the output of public goods: theory and application to state-local finance in the United States", Public Finance, vol. 25, $\mathrm{N}^{\circ} 2$.

Milligan, K. y M. Smart (2005), "Regional grants as pork barrel politics", CEsifo Working Paper, $\mathrm{N}^{\circ} 1453$, Munich, CEsifo Group Munich.

Musgrave, R. (1958), The Theory of Public Finance, Nueva York, McGraw-Hill.

Oates, W. (1972), Fiscal Federalism, Nueva York, Harcourt Brace Jovanovich.

Olson, M. (1965), The Logic of Collective Action, Cambridge, Harvard University Press.

Porto, A. y P. Sanguinetti (2001), "Political determinants of intergovernmental grants: evidence from Argentina", Economics and Politics, vol. 13, $\mathrm{N}^{\circ} 3$, Wiley Blackwell.

Reading, D.C. (1973), "New deal activity and the states, 1933 to 1939”, Journal of Economic History, vol. 33, № 4, Cambridge, Cambridge University Press.

Rogoff, K. (1990), "Equilibrium political budget cycles", American Economic Review, vol. 80, No 1, Nashville, Tennessee, American Economic Association.

Rogoff, K. y A. Sibert (1988), "Elections and macroeconomic policy cycles", Review of Economic Studies, vol. 55, No 1, Oxford, Oxford University Press.

Segura-Ubiergo, A. (2007), "Political instability, regime change, and late economic reform in non-welfare state", The Political Economy of the Welfare State in Latin America. Globalization, Democracy and Developement, Washington, D.C., Fondo Monetario Internacional.

Schady, N.R. (2000), "The political economy of expenditures by the Peruvian social fund (FONCODES) 1991-95", American Political Science Review, vol. 94, N ${ }^{\circ}$ 2, Washington, D.C., American Political Science Association.

Solé-Ollé, A. y P. Sorribas (2008), "The effects of partisan alignment on the allocation of intergovernmental transfers. Differences-indifferences estimates for Spain", Journal of Public Economics, vol. 92, $\mathrm{N}^{\mathrm{o}} 12$, Amsterdam, Elsevier.

Tanaka, Martín (2002), "La dinámica de los actores regionales y el proceso de descentralización: ¿el despertar del letargo?”, Documento de trabajo, No 125 Lima, Instituto de Estudios Peruanos.

Vega, J.C. (2008), "Análisis del proceso de descentralización fiscal en el Perú", Documento de trabajo, No 266, Lima, Pontificia Universidad Católica del Perú.

Wallis, J. (1998), "The political economy of new deal spending revisited, again: with and without Nevada", Explorations in Economic History, vol. 35, 으 2, Amsterdam, Elsevier.

Wright, G. (1974), "The political economy of new deal spending, an econometric analysis", The Review of Economics and Statistics, vol. 56, No 1, Cambridge, Massachusetts, The MIT Press. 